\title{
Aspects diphasiques des échanges particulaires à l'interface air-mer
}

\author{
Etude d'une instrumentation
}

Two-phase aspects of particle-transfert at the air/sea interface

François Resch ${ }^{*}$

Chargé de Recherche au C.N.R.S.
François Avellan*

Ingénieur E.N.S.H.G.
L'étude du mécanisme des échanges particulaires à l'interface air-mer doit être replacée dans le contexte beaucoup plus général de l'étude de la pollution marine qui est, à l'heure actuelle, un problème particulièrement important (par exemple, programmes C.N.E.X.O. en France et SEAREX aux Etats-Unis). Nos études concernent plus spécifiquement les mécanismes par lesquels ces échanges s'effectuent à l'interface marine. Pour donner un exemple de l'importance du phénomène, on évalue ce transfert de matière à $10^{9}$ tonnes par an pour toute la surface des océans.

C'est la production d'aérosols d'origine marine qui constitue la principale source de cet échange de masse. Cette production qui résulte d'un mécanisme "en chaine" peut être schématisée de la façon suivante (voir Fig. 1) :

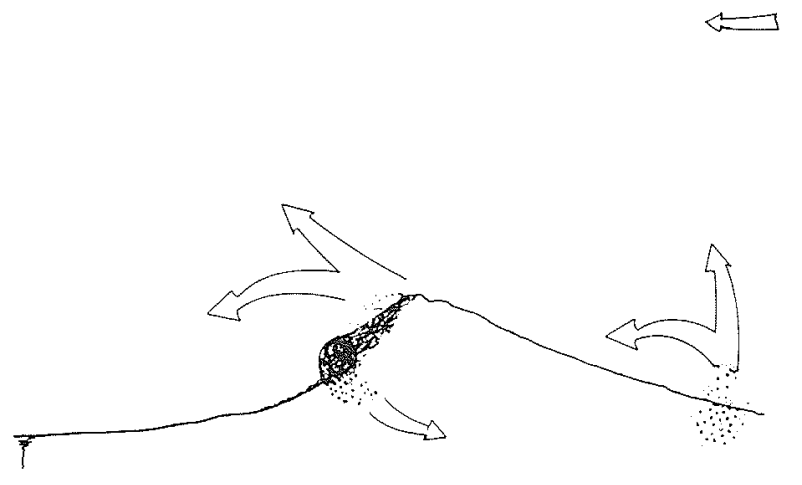

Figure 1 -

(*) Institut de Mécanique Statistique de la Turbulence, Laboratoire associé au C.N.R.S. $n^{\circ} 130,12$, avenue du Général Leclerc - 13003 Marseille.

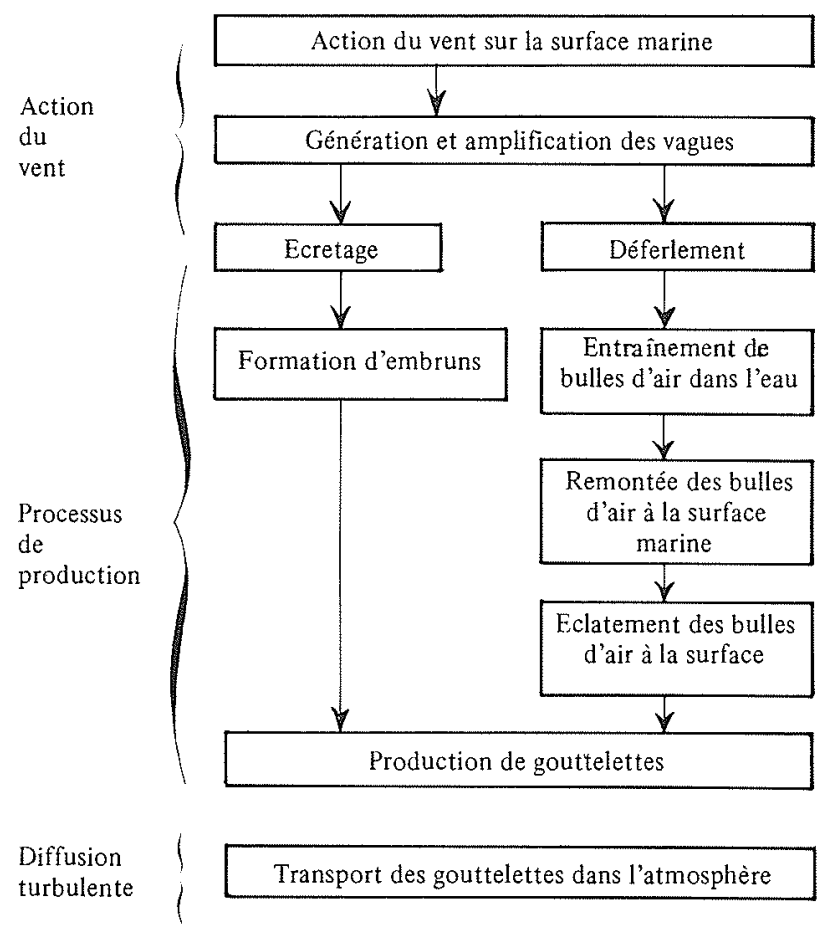

L'effet du vent sur la surface marine est à l'origine du mécanisme de formation des aérosols. Par exemple, Woodcock [1] a trouvé que la concentration en poids de sel marin dans les nuages à basse altitude était augmentée par un facteur de 10 quand la force du vent passait de 3 à 7 . Lorsque la vitesse du vent augmente, le processus d'amplification se poursuit jusqu'à l'apparition du déferlement. En effet, ce dernier apparait quand la vitesse absolue (croissante) des particules 
devient égale à la vitesse de l'ondulation [2]. C'est autour de la valeur critique de la cambrure de la vague que se produisent simultanément les phénomènes d'arrachement de particules liquides de la crête (écrétage) et de formation d'un "rouleau" ou "mousse" (déferlement) entrainant des bulles d'air dans la masse liquide. Ce dernier phénomène est semblable à celui engendré par un ressaut hydraulique [4].

L'entrainement des bulles d'air sera caractérisé par sa profondeur d'injection, sa granulométrie et sa concentration ; ces paramètres étant directement liés à la structure du déferlement, donc à la vitesse du vent.

La trajectographie des bulles d'air dans l'eau est im. portante car l'âge d'une bulle, c'est-à-dire son temps de séjour dans le liquide avant d'atteindre la surface, influe plus ou moins directement sur l'évolution de son diamètre et de la composition chimique de son interface. Ces caractéristiques, à leur tour, sont prépondérantes dans la génération des gouttes lors de l'éclatement de la bulle à la surface.

Les granulométries de bulles d'air, que l'on doit considérer se situent entre 50 microns et 5 millimètres, les vitesses ascensionnelles étant de l'ordre de $10 \mathrm{~cm} / \mathrm{s}$ pour des bulles de $1 \mathrm{~mm}$ de diamètre ou de $0,3 \mathrm{~cm} / \mathrm{s}$ pour des bulles de 100 microns de diamètre.

En atteignant la surface, la plupart des bulles d'air "éclatent" et donnent naissance à la formation de deux familles de gouttes:

1) Les gouttes de film ("film-drops") obtenues par rupture du film interfacial. Cette rupture engendre, d'une part une production horizontale de fines gouttelettes de quelques microns de diamètre dotées d'une vitesse horizontale de l'ordre de $8 \mathrm{~m} / \mathrm{s}$, d'autre part une production verticale de gouttelettes par arrachement du film par l'air qui s'échappe de la bulle (projection en forme de calotte ou de champignon), les gouttes ainsi créées ont également des tailles de l'ordre du micron.

2) Les gouttes de jet ("jet-drops") : la remontée du fond de la bulle provoque une éjection de quelques (3 à 5) gouttes d'eau à des hauteurs variables. Les accélérations mises en jeu par ce phénomène sont très fortes : de l'ordre de $10^{6} \mathrm{~g}$ pour des bulles de 10 microns de diamètre et de $10^{3} \mathrm{~g}$ pour des bulles de $1 \mathrm{~mm}$. Les hauteurs d'éjection (dans des conditions normales propres - de surface) sont de l'ordre de $20 \mathrm{~cm}$ pour la première goutte et inférieures à $5 \mathrm{~cm}$ pour les gouttes suivantes (cet ordre de grandeur est donné à titre indicatif pour une bulle d'air de $2 \mathrm{~mm}$ de diamètre). En ce qui concerne la granulométrie des gouttes de jet, on considère en général leur diamètre comme approximativement égal à 0,15 fois le diamètre de la bulle d'air génératrice, soit de quelques microns à 500 microns.

Notre but est donc de déterminer les paramètres diphasiques caractéristiques suivants:

- granulométrie - concentration - profondeur d'injection pour les bulles d'air entrainées dans l'eau [3] ;

- granulométrie - concentration - hauteur d'éjection pour les gouttes d'eau obtenues par éclatement des bulles d'air à la surface et de les corréler aux conditions dynamiques de l'écoulement d'air. Cette étude sera effectuée dans la grande soufflerie de simulation des interactions océans-atmosphère de l'I.M. S.T. Le premier stade de l'étude expérimentale de ce sujet est de définir, mettre au point et étalonner une instrumentation adaptée aux exigences mentionnées ci-dessus.

La gamme des diamètres rencontrés s'étend donc, dans l'eau, de quelques dizaines de microns à quelques millimètres et, dans l'air, de quelques microns à quelques centaines de microns.

Dans l'élaboration des critères qui permettent de déterminer les procédés de mesure susceptibles de répondre à nos besoins, nous devrons aussi tenir compte de la très fine dispersion des écoulements dans l'air et dans l'eau, hors de la zone de déferlement : moins d'une centaine de particules par centimètre cube $[5$, $6]$.

Parmi les procédés existants déjà on peut schématiquement distinguer quatre grandes familles :

- les procédés de mesures électriques par sonde ou par mesure des mobilités électriques ; mais celles-ci ne couvrent pas notre gamme de diamètres; - les procédés de mesures acoustiques reposant sur le phénomène de résonance, utilisé en particulier par Medwin [7] qui a mesuré dans l'océan des bulles de 15 à 300 microns : ces procédés ne semblent pas être utilisables dans l'air ;

-- les procédés de mesures par microscopie qui nécessitent la captation préalable des particules sur un support : mais celles-ci ne permettent pas un traitement automatique des données ;

- les procédés de mesures photoélectriques utilisant les lois de la diffusion de la lumière : ce sont ceux que nous avons retenus parce qu'ils sont susceptibles d'être mis en oeuvre de part et d'autre de l'interface dans un milieu très dispersé pour couvrir la gamme des diamètres décrite ci-dessus, tout en permettant un traitement automatique des données.

Distinguons les mesures globales qui fournissent directement une concentration, des mesures locales qui donnent des granulométries relatives.

\section{Procédés de mesure globale}

Parmi les procédés qui permettent des mesures globales, nous avons éliminé les techniques holographiques à cause de la lenteur du dépouillement et de l'investissement nécessaire.

Nous avons retenu deux méthodes : l'une qui consiste à analyser les images de diffraction à l'infini des particules, l'autre à mesurer l'atténuation d'un faisceau lumineux traversant l'écoulement.

\section{Méthode de diffraction à l'infini}

Pour analyser l'image de diffraction de l'ensemble des particules éclairées par un faisceau cohérent col- 


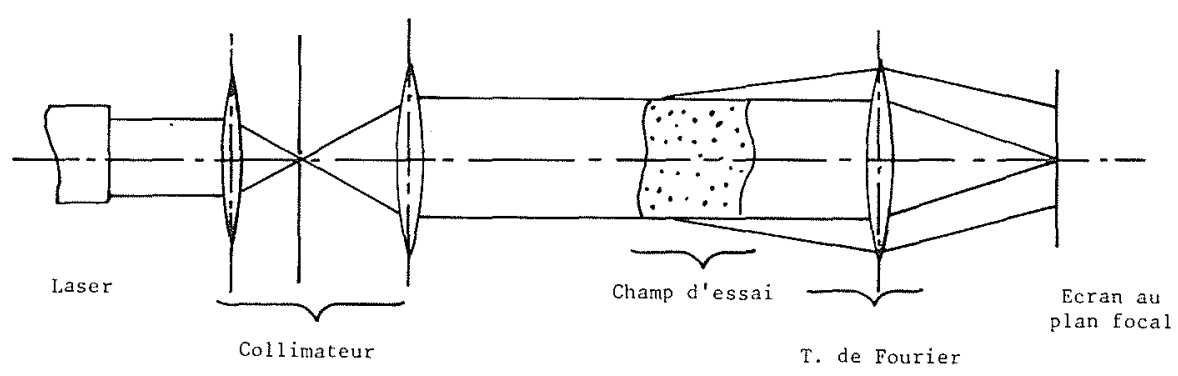

Figure 2 -

limaté, on se place au plan focal d'une lentille qui effectue en réalité la transformée de Fourier de l'image de la dispersion ; le montage de principe est indiqué sur la figure 2. Si les particules sont sphériques, la formule de Bouguer-Beer permet de relier la granulométrie de la dispersion à la répartition de l'intensité lumineuse sur la figure de diffraction [8]. Toute la difficulté réside dans la mesure de cette répartition : on peut, soit disposer un diaphragme variable devant un photorécepteur, soit utiliser un micro-densitomètre se déplaçant dans le plan focal. Notons qu'il existe un développement commercial( $\left.{ }^{1}\right)$ d'une telle méthode avec un photodétecteur composé d'une trentaine de photodiodes annulaires. Ce procédé comporte cependant une restriction en ce qui concerne la dimension de l'optique de reprise qui limite l'étendue du champ de mesure.

\section{Méthodes d'atténuation}

Cette méthode consiste à mesurer la fraction de lumière parallèle transmise à travers la dispersion. D'une manière générale, la fraction de lumière transmise qui est le rapport de l'intensité reçue $I$ à l'intensité $I_{o}$ du faisceau incident, s'exprime par :

$$
I / I_{o}=\exp \{-\gamma . l\}
$$

où $l$ est la longueur du chemin optique et $\gamma$ le coefficient d'atténuation. La détermination de ce coefficient est fonction de la granulométrie de la dispersion. Dans le cas particulier où les diamètres sont grands par rapport à la longueur d'onde de la lumière, McLaughin et Rushton [9] ont trouvé que $\gamma$ était proportionnel à la surface spécifique quelle que soit la forme du spectre dimensionnel de la dispersion. Le principe d'une telle méthode est schématisé sur la figure 3.

(1) ST 1800 Malvern Instruments Ltd.
Notons la nécessité de connaitre le rapport $I / I_{o}$ tout en s'affranchissant des fluctuations de la source et en ayant soin d'obtenir une répartition uniforme d'intensité dans une section droite du faisceau de lumière.

Si le nombre de particules est petit, on peut réduire la section du faisceau afin que la probabilité d'avoir plus d'une particule "éclairée" soit faible. J. Wu [10] a développé une telle technique en utilisant un double faisceau laser : il a obtenu les diamètres des particules et leurs vitesses tout en tenant compte de la répartition gaussienne de l'intensité lumineuse dans le faisceau. Bien que les diamètres qu'il ait pu mesurer soient supérieurs à 30 microns, il semble possible d'abaisser cette limite eń améliorant le montage optique, notamment par l'adjonction d'un filtre spatial tel qu'il est indiqué sur la figure 3 .

\section{Procédés de mesure locale}

Citons tout d'abord un procédé développé par Knollenberg [11] qui analyse l'image géométrique de l'ombre de la particule par un réseau de fibres optiques reliées à un photodétecteur ; cette méthode est limitée au domaine de l'optique géométrique, c'est-à-dire à des diamètres supérieurs à 10 microns. La plupart des procédés optiques de mesures locales consistent à évaluer la quantité de lumière diffusée par une particule supposée sphérique.

En effet, la théorie de Mie dont on trouvera une description dans l'ouvrage de Hulst [12] montre qu'une particule soumise à une onde incidente, diffuse dans toutes les directions.

La loi de répartition de l'intensité diffusée dans l'espace a été calculée pour une certaine gamme de diamètres et d'indices de réfraction (voir, par exemple, Gumprecht [13]).

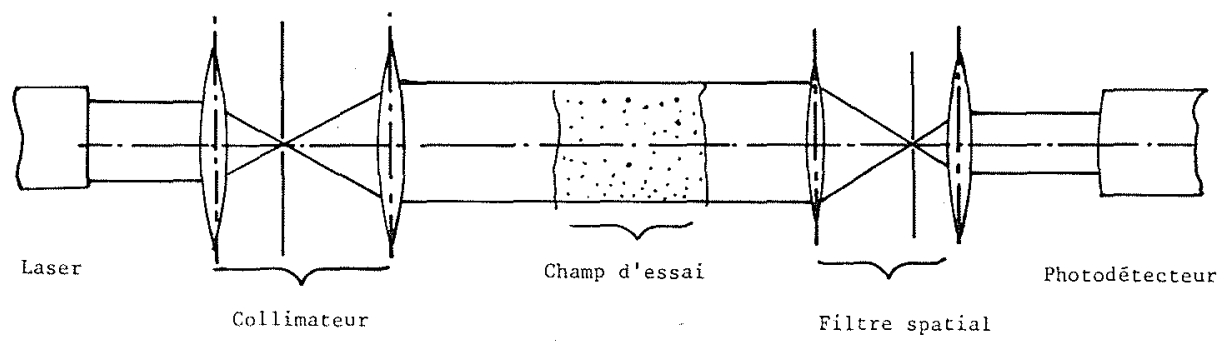

Figure 3 - 


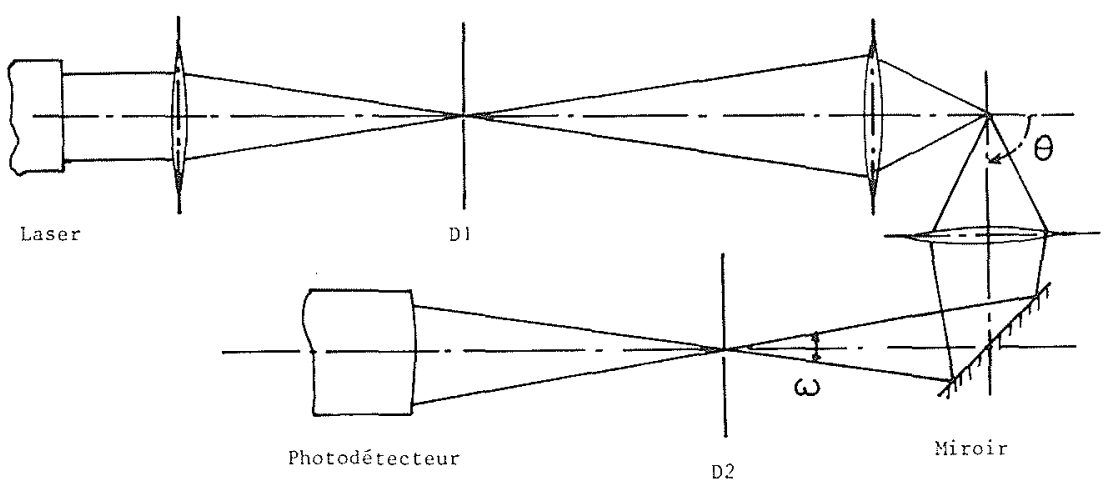

Figure 4

Pour déterminer le diamètre d'une particule d'indice connu il suffit alors, soit de mesurer l'intensité lumineuse dans plusieurs directions, soit de mesurer le flux diffusé sous un certain angle dans une direction donnée. Ederhof [14] a développé une telle technique en mesurant le flux pour une ouverture donnée sous une incidence de $90^{\circ}$. Notons que, suivant le même principe, il a été mis au point par la Météorologie Nationale une sonde $\left({ }^{2}\right)$ utilisable à partir d'un avion et qui mesure le flux diffusé sous une incidence de $30^{\circ}$, couvrant par ailleurs une gamme de 5 à 300 microns.

$\mathrm{Si}$ on se reporte au montage de principe indiqué sur la figure 4 , on voit que le volume de mesure est délimité par les images des diaphragmes $D_{1}$ et $D_{2} ;$ ceci permet, en ajustant l'ouverture de ces derniers, de contrôler les dimensions du domaine sensible. Afin d'avoir un volume "net", c'est-à-dire pour éviter les effets de bord, il est nécessaire d'avoir un éclairement uniforme des diaphragmes tout en évitant des diffractions parasites sur les contours de ces derniers. A cette difficulté de réalisation vient s'ajouter la sensibilité du flux diffusé aux variations d'indice de réfraction des particules (Turpin, [15]).

\section{Conclusions}

En résumé, nous avons sélectionné :

- pour les mesures locales, le procédé de diffusion à $90^{\circ}$;

- pour les mesures globales, le procédé d'atténuation.

Ces méthodes indirectes de mesure nécessitent une mise au point et un étalonnage précis. Dans ce but, un banc d'étalonnage sera spécialement réalisé. Il permettra par ailleurs d'effectuer des recoupements entre les résultats des mesures locales et ceux des mesures globales.

\section{Remerciements}

Cette note a été effectuée dans le cadre du contrat CNEXO/IMST n” 77/1673 : "Etude des mécanismes des échanges de masse à travers l'interface air-mer, sous forme gazeuse et particulaire".

(2) Aquasonde

\section{Bibliographie}

[1] BLANCHARD D.C., WOODCOCK A.H. - Bubble formation and modification in the sea and its meteorological significance. Tellus, vol. $9, \mathrm{n}^{\circ} 2,1957$.

[2] LACOMBE H. - Cours d'océanographie physique. Ed. Gauthier-Villars, Paris, 1965.

[3] RESCH F.J., LEUTHEUSSER H.J., ALEMU S. - Bubbly Two-Phase flow in hydraulic jump. Journal of the Hydraulics Division, A.S.G.E. vol. 100 n$^{\circ}$ HY 1, Proc. Paper 10297 , pp. 137-149, 1974.

[4] RESCH F.J., LEUTHEUSSER H.J., COANTIC M. - Etude de la structure cinématique et dynamique du ressaut hydraulique. Journal of Hydraulic Research, vol. 14, $\mathrm{n}^{\circ} 4, \mathrm{pp} .293$ 319,1976

1976.

[5] CHAEN M. - Studies on the production of sea salt particles on the sea surface. Memoirs of the Faculty of Fisheries, Kagoshima University, vol. 22, n 2,1973

[6] KOLOVAYEV P.A. - Investigation of the concentration and statistic size distribution of wind-produced bubbles in the near surface ocean layer. Okeanology, 15, pp. 659-661, 1975.

[7] MEDWIN H. - In situ acoustic measurements of microbubbles at sea : Joumal of Geophysical Research, vol. 82, $\mathrm{n}^{\circ} 8$, pp. 971-976, 1977

[8] CHIN J.H., SLIEPCEVICH C.M., TRIBUS M. - Particle size distributions from angular variation of intensity of forward-scattered light at very small angles. Journal of Physical Chemistry, 59, pp. 841-844, 1955

[9] McLAUGHIN C.G., RUSHTON J.H. - Interfacial areas of liquid dispersions from hight transmission measurements Journal of A.I.Ch.E., 19, n ${ }^{\circ} 4$, pp. 817-822, 1973.

[10] WU J. - Fast moving suspended particles : measurements of their size and velocity. Applied Optics, $16 \mathrm{n}^{\circ} 3$, pp. 596600,1977

[11] KNOLLENBERG R.G. - The optical array : an alternative to scattering or extinction for airborne particle size determination. Journal of Applied Meteorology, 9, $\mathrm{n}^{\circ}$ 1, pp. 86-103, 1970.

[12] Van der HULST H.C. - Light scattering by small particles. Wiley and sons, New York, 1957.

[13] GUMPRECHT R.O., SUNG N.L., CHIN J.H., SLIEPCECICH C.M. - Angular distribution of intensity of light scattered by large droplets of water. Journal of the optical Society of America, 42, ${ }^{\circ} 4$, pp. 226-231.

[14] EDERHOF A. - A light-scattering probe for droplet size and wetness fraction measurement in two-phase flows. Two-Phase steam flow in Turbines and separators. Ed. Moore M.J., Sieverding C.H., Hemisphere publishing Corp. London, pp. 249-260, 1976

[15] TURPIN P.Y., REISS P., BRICARD J., MADELAINE G. Nouveaux procédés photoélectriques de mesure des aérosols. Revue générale de Thermique, $12, \mathrm{n}^{\circ} 142, \mathrm{pp} .937$ 952,1973 